\title{
Detection of extended spectrum beta-lactamase producing Gram-negative organisms: hospital prevalence and comparison of double disc synergy and E-test methods
}

\author{
Mili Rani Saha and Sanya Tahmina Jhora \\ Department of Microbiology, Sir Salimullah Medical College, Mitford, Dhaka, Bangladesh
}

\begin{abstract}
Background and objectives: Emergence of extended spectrum beta-lactamase (ESBL) producing bacteria is a major public health concern. Detection of multi drug resistant (MDR) ESBL producing organisms is necessary to prevent its spread and effective treatment. The purpose of the present study was to determine the magnitude of ESBL producing organism in hospital setting and to compare the suitability of double disc synergy test (DDST) and cefepime-clavulanate E-test method for the detection of ESBL producing organisms in routine microbiology laboratory.

Materials and methods: The study was carried out in the Department of Microbiology, Sir Salimullah Medical College, Dhaka from January 2011 to December 2011. Clinical samples included urine and pus from patients with suspected urinary tract and wound infections respectively. Standard microbiological methods were employed for isolation and identification of the organisms. DDST and E-test were used to detect ESBL producing Gram negative organisms.

Results: A total of 186 Gram-negative organisms were isolated from various samples. Among the 186 Gram negative bacteria, 120 (64.5\%) were Esch. coli while 33 (17.7\%), 20 (10.8\%) and 11 (5.9\%) were Pseudomonas sp, Klebsiella sp and Proteus sp respectively. Out of total 186 isolates, $77(41.4 \%)$ and $73(39.2 \%)$ isolates were found ESBL producers by DDST and E-test method $(\mathrm{p}=0.674)$ respectively. Compared to Escherichia coli, Pseudomonas and Proteus, significantly high $(\mathrm{p}<0.01)$ proportion of Klebsiella were ESBL positive by both DDST and E-test methods. The detection rate of ESBL producing organisms was not significantly different by DDST and E-test $(41.4 \%$ vs $39.2 \%)$. Non-determinable result was obtained for $4(2.2 \%)$ isolates by E-test method.

Conclusion: In our present study, a substantially large number of clinical isolates were found ESBL producers. Compared to E-test, DDST was found as a reliable, convenient and inexpensive method for detection of ESBL producing organism in routine microbiology laboratory practice.
\end{abstract}

IMC J Med Sci 2018; 12(1): 32-36

\section{Introduction}

Extended spectrum beta-lactamases are enzymes that confer resistance to the penicillin, cephalosporins and aztreonam by hydrolysis of the antibiotics. ESBL enzymes are inactivated by beta-lactamase inhibitors such as clavulanic acid [1]. Treatment of
ESBL producing organisms is now a therapeutic challenge in hospitalized patients worldwide. Indiscriminate administration of extended spectrum cephalosporins, prolonged hospital stay, mechanical ventilation and catheterization are the major risk factors for colonization of ESBL producing

\footnotetext{
Address for Correspondence:

Dr. Mili Rani Saha, Assistant Professor, Department of Microbiology, BIRDEM General Hospital, 122, Kazi Nazrul Islam Avenue, Dhaka 1000,Bangladesh,Email: milisaha77@yahoo.com
} 
bacteria [2]. Detection of ESBL producing organisms is necessary to prevent its spread. Several ESBL detection tests have been proposed by NCCLS [3]. The degree of resistance against extended spectrum cephalosporins can also be highly variable for the different ESBL enzymes. Thus, ESBL producing bacteria need reliable detection method [4].

Double Disc Synergy Test (DDST), is a sensitive, convenient and inexpensive method for detection of ESBL producing organisms in routine clinical laboratory service. In DDST, easily available commercial antibiotic sensitivity discs are used. Sensitivity and specificity of the method range from $79 \%$ to $97 \%$ and $94 \%$ to $100 \%$ respectively [5]. Recommendations of the Clinical and Laboratory Standards Institute (CLSI) for phenotypic confirmation of ESBL still rely on the minimum inhibitory concentration (MIC) difference test, in which a $\beta$-lactamase inhibitor is used to protect the activity of an indicator drug against an ESBL producing strain [6]. Apart from DDST, commercially available E-test is technically straight forward, versatile and easy to use method for ESBL detection and is an attractive alternative to conventional MIC dilution test. Three types of E-test strips such as cefepime-clavulanate, cefotaxime-clavulanate and ceftazidime-clavulanate in different antibiotic gradient concentrations are available for the detection of ESBL. Detection of MIC using E-test is convenient and easy to use, but it is sometime difficult to read the test when the MIC of the bacterial strain are below or above the range of antibiotics used in the strip [7]. Therefore, the result may be non-determinable if the MIC of the test organism is beyond the antibiotic range [8]. Sensitivity and specificity of E-test for detection of ESBL producers are $87 \%$ to $100 \%$ and $95 \%$ to $100 \%$ respectively [9]. Moreover, E-test strip method is expensive and gives false negative result, which may cause therapeutic failure. Except in a few microbiology laboratories, ESBL screening is not routinely practiced in Bangladesh.

Therefore, the present study was undertaken to determine the magnitude of ESBL producing organisms in tertiary hospitals and to compare the DDST with that of commercially available E-test to detect ESBL producing Gram negative organisms isolated from clinical samples.
Materials and Methods

Study population and place: This Cross-sectional study was carried out at the Department of Microbiology, Sir Salimullah Medical College, Dhaka for a period of one year from January 2011 to December 2011. A total 354 clinical samples were collected from inpatient and outpatient departments of Mitford Hospital, and Burn Unit of Dhaka Medical College Hospital in Dhaka. Samples were collected from suspected cases of urinary tract infection, burn and surgical wound infections.

Isolation and identification of organisms: Samples were inoculated onto blood agar and MacConkey's agar media for isolation and identification of the organisms. All plates were incubated at $37^{\circ} \mathrm{C}$ aerobically for 24-48 hrs. Suspected organisms were identified by standard biochemical tests [10].

Tests for the detection of ESBL producing organisms: All isolated organisms were tested for ESBL production by DDS test and E-test methods.

a. Double Disc Synergy Test: All isolated Gram-negative bacteria were tested for ESBL production by DDST using aztreonam (30 $\mu \mathrm{g})$, ceftazidime $(30 \mu \mathrm{g})$, ceftriaxone $(30 \mu \mathrm{g})$, cefotaxime $(30 \mu \mathrm{g})$ and $20 \mu \mathrm{g}$ amoxicillin $+10 \mu \mathrm{g}$ clavulanic acid discs. The four antibiotic discs were placed $20 \mathrm{~mm}$ apart from each other with amoxicillin /clavulanic acid disc at the center as shown in Fig-1a. ESBL production was considered positive when the zone of inhibition around any antibiotic disc was enhanced towards the amoxicillin/clavulanic acid disc [11].

b. Detection of ESBL by E-test Method: E-test cefepime/cefepime+clavulanic acid (PM / PML) strip (AB Biodisk, Solna, Sweden), containing cefepime / cefepime+clavulanic acid (cefepime MIC range, 0.25-16 mg/L; Cefepime/clavulanic acid MIC range 0.064-4 $\mathrm{mg} / \mathrm{L}$ plus $4 \mathrm{mg} / \mathrm{L}$ clavulanic acid) were used. E-test procedure, reading and interpretation were carried out according to the manufacturer's instructions. The presence of ESBL is confirmed by the appearance of a phantom zone or deformation of the PM ellipse (Fig-1b) or when the MIC of PM has been reduced by $\geq 3$ two-fold dilutions in 
presence of clavulanic acid at PML side. Klebsiella pneumoniae ATCC 700603 and Escherichia coli ATCC 25922 were used as positive and negative control.

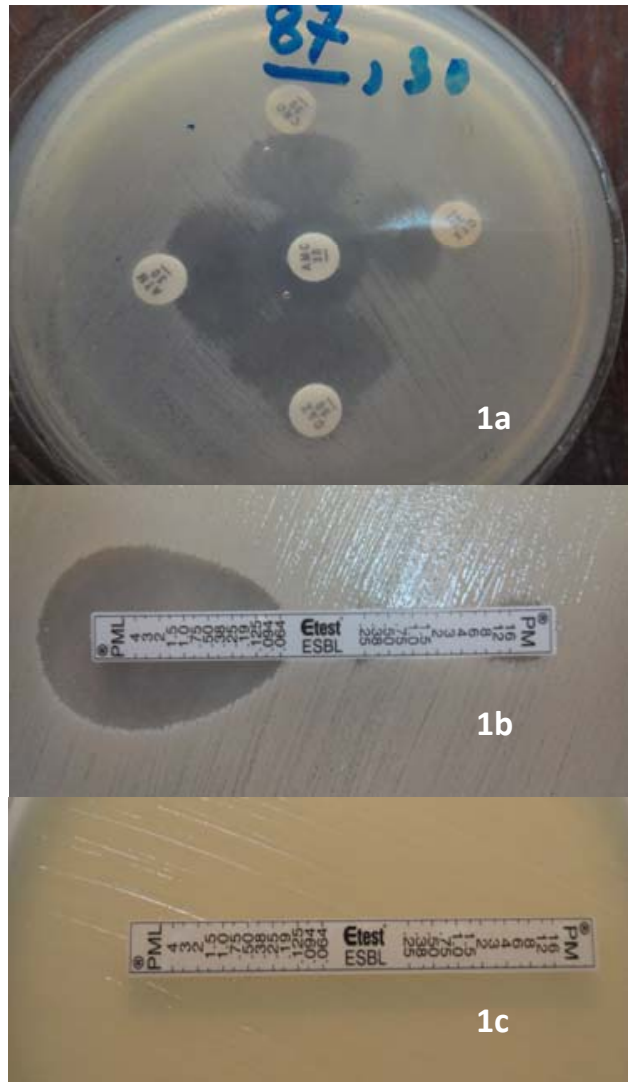

Fig.1: Photograph showing DDST and E-test. 1a: DDST showing enhancement of zone of inhibition towards the aztreonam $(30 \mu \mathrm{g})$, ceftazidime $(30 \mu \mathrm{g})$, ceftriaxone $(30 \mu g)$ and cefotaxime $(30 \mu g)$ discs. Amoxicillin/clavulanic acid disc is at the center. $1 b$ : E-test strip showing formation ellipse at the cefepime/clavulanic end compared to on cefepime end; 1c: E-test with non-determinable result.

\section{Results}

A total of 354 samples were included in the study. Total 186 Gram-negative organisms were isolated from various samples. Among the 186 Gram negative bacteria, 120 (64.5\%) were Escherichia coli while 33 (17.7\%), 20 (10.8\%) and 11 (5.9\%) were Pseudomonas sp, Klebsiella sp and Proteus sp respectively. All the isolates were tested for production of ESBL by DDST and E-test methods. Out of total 186 isolates, $77(41.4 \%)$ and 73 $(39.2 \%)$ isolates were found ESBL producers by DDST and E-test method $(p=0.674)$ respectively (Table-I). Compared to Escherichia coli, Pseudomonas and Proteus species, significantly high $(\mathrm{p}<0.01)$ proportion of Klebsiella sp $(65-$ $75 \%$ ) were ESBL positive by both DDST and Etest methods (Table-1). The detection rate of ESBL was not significantly different by DDST and E-test for each type of organism. Non-determinable result was obtained in 4 isolates by E-test method. These four isolates did not show any zone of inhibition either at cefepime (PM) or at cefepimeclavulanate (PML) end of the test strip (Fig 1c).

Table-1: Comparative detection rate of ESBL producing organisms by DDST and E-test

\begin{tabular}{lcccc}
\hline \multirow{2}{*}{ Organism } & \multirow{2}{*}{$\begin{array}{c}\text { Total } \\
\text { Number }\end{array}$} & \multicolumn{2}{c}{ ESBL positive by } & E-test \\
\cline { 3 - 4 } & & DDST & E-test & ND \\
& & N $(\%)$ & N (\%) \\
\hline Esch. coli & 120 & $50(41.7)$ & $48(40.0)$ & $2(1.7)$ \\
Pseudomonas spp. & 33 & $7(21.2)$ & $7(21.2)$ & 0 \\
Klebsiella spp. & 20 & $15(75.0)$ & $13(65.0)$ & $2(10.0)$ \\
Proteus spp. & 11 & $5(45.5)$ & $5(45.5)$ & 0 \\
Total & $\mathbf{1 8 6}$ & $\mathbf{7 7}(\mathbf{4 1 . 4 )}$ & $\mathbf{7 3 ( 3 9 . 2 )}$ & $\mathbf{4 ( 2 . 2 )}$ \\
\hline
\end{tabular}

Note: $D D S T=$ Double disc synergy test;

$$
N D=\text { Non-determinable } \text {. }
$$

\section{Discussion}

In recent years, beta-lactamase producing Gramnegative bacilli have increased and become a leading cause of resistance to beta-lactam antibiotics [10]. Detection of ESBL producing strains is important for instituting effective treatment and containment of its outbreak in hospitals and healthcare facilities [12]. The present study has revealed that a large number of Gramnegative organisms were ESBL producers imparting a major threat in escalation of treatment cost and spread of these multi drug resistant organisms. ESBL producing organisms were detected with equal efficiency by both DDST and E-test methods. However, four isolates which were found as ESBL producers by DDST were nondeterminable by E-test. Probably, the MICs of those isolates to cefepime were beyond the 
cefepime concentration present in the PM/PML Etest strip (cefepime MIC range 0.25-16 mg/L; Cefepime/clavulanic acid MIC range 0.064-4 $\mathrm{mg} / \mathrm{L}$ plus $4 \mathrm{mg} / \mathrm{L}$ clavulanic acid) [13]. It appears that the DDST could be more sensitive in detecting potential ESBL producers as it takes into account the enhancement or potentiation of zone of inhibition against any of the four antibiotics discs namely ceftriaxone, ceftazidime, cefotaxime and aztreonam [14,15]. However, such discrepant results should be confirmed by molecular methods or by wide range MIC test.

The study has demonstrated that a good proportion of Gram-negative organisms isolated from clinical samples were ESBL producers. Compared to expensive E-test, DDST is a reliable, convenient, relatively inexpensive and easy to perform method for detection of ESBL producing organisms in routine clinical laboratories.

\section{Author's contributions}

MRS performed the experiments analyzed the results and wrote the manuscript. STJ conceived, designed and supervised the study.

\section{Competing interest}

Authors declare no conflict of interest.

\section{Funding}

None

\section{References}

1. Paterson DL, Bonomo RA. Extended-S.pectrum $\beta$-Lactamases: a Clinical Update. Clin Microbiol Rev. 2005; 18(4): 657-686.

2. Chaudhary U, Aggarwal R. Extended spectrum $\beta$-lactamases (ESBL) - An emerging threat to clinical therapeutics. Indian $\mathrm{J} \mathrm{Med}$ Microbiol. 2004; 22(2): 75-80.

3. Clinical and Laboratory Standards Institute. Performance Standards for Antimicrobial Disk Susceptibility Tests; Approved Standard. Clinical and Laboratory Standards Institute Document M100-S9. PA, USA, 1999.

4. Sturenburg E, Mack D. Extended spectrum $\beta$ lactamases: implications for the clinical microbiology laboratory, therapy and infection control. J Infect. 2003; 47: 273-295.

5. Paterson DL, Bonomo RA. ExtendedSpectrum $\beta$-Lactamases: a clinical update. Clin Microbiol Rev. 2005; 18(4): 657-686.

6. National Committee for Clinical Laboratory Standards. Performance standards for antimicrobial susceptibility testing. Twelfth informational supplement. M100 -S12 NCCL. PA, USA, 2002.

7. Bradford PA. Extended spectrum betalactamases in 21st century: characterization, epidemiology, and detection of this important resistance threat. Clin Microbiol Rev. 2001; 14(4): 933-951.

8. d'Azevedo PA, Gonçalves AL, Ramos CG, Dias CA. Laboratory tests in the detection of extended spectrum beta-lactamase production: National Committee for Clinical Laboratory Standards (NCCLS) screening test, the E-test, the double disk confirmatory test, and cefoxitin susceptibility testing. Braz J Infect Dis. 2004; 8(5): 372-377.

9. Jain A, Mondal R. TEM \& SHV genes in extended spectrum $\beta$-lactamase producing Klebsiella species \& their antimicrobial resistance pattern. Indian J Med Res. 2008; 128: 759-764.

10. Colle JG, Miles RS, Watt B. Tests for identification of Bacteria. In: Collee JG, Fraser AG, Marmion BP \& Simmons A. editors, Mackie \& McCartney's Practical Medical Microbiology 1996; 14th ed and Edinburgh, Churchill Livingstone, New York 1996; $553-559$.

11. Jarlier V, Nicolas MH, Fourier G, Phillippon A. Extended broad spectrum $\beta$-lactamases conferring transferable resistance to newer $\beta$ lactam agents in Enterobacteriaceae: hospital prevalence and susceptibility patterns. Rev Infect Dis. 1988; 10: 867-878.

12. Rodrigues C, Joshi P, Jani SH, Alphonse M, Radhakrishnan R, Mehta A. Detection of betalactamases in nosocomial Gram-negative clinical isolates. Indian $J$ Med Microbiol. 2004; 22: 247- 250. 
13. Livermore DM. $\beta$-lactamases-mediated resistance and opportunities for its control. J Antimicrob Chemother. 1998; 41 suppl D: 25-41.

14. Vercauteren E, Descheemaeker P, Ieven M, Sanders CC, Goossens H. Comparison of screening methods for detection of extendedspectrum $\beta$-lactamases and their prevalence among blood isolates of Escherichia coli and
Klebsiella spp. in a Belgian teaching hospital. J Clin Microbiol. 1997; 35: 2191-2197.

15. Wiegand I, Geiss HK, Mack D, Sturenburg E, Seifert H. Detection of extended-spectrum $\beta$ lactamases among Enterobacteriaceae by use of semiautomated microbiology systems and manual detection procedures. J Clin Microbiol. 2007; 45: 1167-1174. 